\title{
Review of: "Impact of intensifying nitrogen limitation on ocean net primary production is fingerprinted by nitrogen isotopes"
}

\author{
Meiling Zhang ${ }^{1}$ \\ 1 Gansu Agricultural University
}

Potential competing interests: The author(s) declared that no potential competing interests exist.

This study performs four simulations using the Pelagic Interactions Scheme for Carbon and Ecosystem Studies version 2 (PISCES-v2) biogeochemical model forced by output from the Institut Pierre-Simon Laplace Climate Model 5A (IPSL-CM5A) and applies a global ocean-biogeochemical model with nitrogen isotopes to investigate the anthropogenic perturbation of the marine nitrogen cycle in the twenty-first century. This study examines the effects of the marine nitrogen cycle on ocean net primary production by analyzing nitrogen isotopes changes, providing an important means to assess model projections of NPP and an enigmatic marine nitrogen cycle. The major finding is that nitrogen isotopes $\square \delta^{15} \mathrm{~N} \square$ changes to nitrogen limitation and NPP over the twenty-first century, experiments demonstrate the dominant role of climate change in the $21^{\text {st }}$ century alteration of the marine nitrogen cycle, and find that nitrogen isotopes fingerprint the dominant role of climate change, specifically circulation changes can intensify nitrogen limitation of lower latitude ecosystems, leading to decrease NPP and nitrogen sinks, and alter the bioavailable nitrogen budget.

There are several innovative points with this study which include the following:

1. This study reveals the impact of two major anthropogenic drivers, atmospheric nitrogen deposition and climate change, on the ocean nitrogen cycle.

2. This study assesses changes in biogeochemical variables related to nitrogen cycling in two depth zones defined by light, the variation of nitrogen isotopes in the marine euphotic zone and the twilight zone is studied.

3. Four initial simulations to quantify the impacts of anthropogenic climate change and nitrogen deposition, to understand the direct and indirect effects of climate change, this study undertakes two additional idealized simulations.

4. The direct and indirect effects of climate change on the nitrogen cycle are measured by Spearman's rank correlation coefficient.

5. To follow nitrogen uptake and fractionation by phytoplankton in a water parcel, this study constructs a zero-dimensional (OD) model; this OD model simulates the nitrogen isotope dynamics in a recently upwelled water parcel during transit to the subtropics.

6. This study's simulations agree with field studies and data-constrained modeling to highlight the 
expected declines in low-latitude NPP associated with increasing nitrogen limitation. 